\title{
A CHARACTERIZATION OF $C^{*}$-SUBALGEBRAS
}

\author{
JAN A. VAN CASTEREN
}

\begin{abstract}
Let $\boldsymbol{A}$ be a closed linear subspace of a $C^{*}$-algebra $B$. Adjoin, if necessary, the identity 1 to $B$. Then $A$ is a $C^{*}$-subalgebra if and only if, for each $x$ in $A$, the elements $x^{*}$ and $|x|+1-|| x|-1|$ are in $A$. If 1 is in $A$, then $A$ is a $C^{*}$-subalgebra if and only if $|x|$ is in $A$ for each $x$ in $A$. Here $|x|$ denotes the unique positive square root of $x^{*} x$ in $B$.
\end{abstract}

This note is entirely devoted to the proof of and a discussion on the following result.

Theorem. Let $A$ be a closed linear subspace of a complex $C^{*}$-algebra $B$. $A$ is $a C^{*}$-subalgebra of $B$ if and only if, for each $x$ in $A$, the elements $x^{*}$ and $|x|+1-|| x|-1|$ are in $A$.

Here $|x|$ denotes the unique positive square root of $x^{*} x$ in $B$. The element $|x|+1-|| x|-1|$ is to be interpreted as $g(|x|)$, where $g(t)=t+1-\mid t-$ $1 \mid, t \geqslant 0$. Since $g(0)=0$, the continuous functional calculus shows that $g(|x|)$ is in $B$; see Proposition 1.5.6, p. 11 of Dixmier [3]. In fact it follows that $|x|+1-|| x|-1|$ is in the $C^{*}$-algebra generated by $x^{*} x$.

Proof. The necessity is included in the above remark.

SUfFICIENCY. Let $x$ be in $A$. The equality

$$
|x|^{2}=\int_{0}^{\infty}\left\{(|x|+t-|| x|-t|)-\left(|x|+\frac{1}{2} t-|| x\left|-\frac{1}{2} t\right|\right)\right\} d t
$$

proves that $x^{*} x=|x|^{2}$ is in $A$. The polarisation formula

$$
4 a^{*} b=|a+b|^{2}-|a-b|^{2}+i\left(|a-i b|^{2}-|a+i b|^{2}\right)
$$

shows that $a^{*} b$ is in $A$ whenever $a$ and $b$ are in $A$. Since $x^{*}$ is in $A$, whenever $x$ is in $A$, we see that $A$ is a $C^{*}$-subalgebra.

Corollary 1. Let $A$ and $B$ be as in the Theorem. If each element of $A$ is normal, then $A$ is a $C^{*}$-subalgebra of $B$ if and only if, for each $x$ in $A$, the element $|x|+1-|| x|-1|$ is in $A$.

Proof. The sufficiency part needs some explanation. Let $x$ be in $A$ and $n$ in N. Since $x^{*} x=x x^{*}$, it follows from the necessity part of the Theorem and from the polar decomposition of $x$ that

Received by the editors April 27, 1977 and, in revised form, August 5, 1977.

AMS (MOS) subject classifications (1970). Primary 46L05, 46A40.

$K e y$ words and phrases. $C^{\star}$-subalgebra, Stone lattice, positive square root.

(- American Mathematical Society 1978 


$$
\begin{aligned}
\| 2 x^{*}-x^{*}(n|x| & +1-|n| x|-1|)\|=\|(|n| x|-1|-n|x|+1) x^{*} \| \\
& =\|(|n| x|-1|-n|x|+1)|x|\| \\
& \leqslant \sup \{(|n t-1|-n t+1) t ; 0 \leqslant t \leqslant\|x\|\} \\
& \leqslant(2 n)^{-1} .
\end{aligned}
$$

So $x^{*}$ is in $A$ by the proof of the sufficiency part of the Theorem.

Corollary 2. Let $A$ and $B$ be as in the Theorem. Assume that $B$ has identity 1. If 1 is in $A$, then $A$ is a $C^{*}$-subalgebra if and only if $|x|$ is in $A$ for each $x$ in A.

Proof. The necessity is clear and the sufficiency follows from the equality

$$
4 x^{*}=|x+1|^{2}-|x-1|^{2}+i\left(|x-i|^{2}-|x+i|^{2}\right), \quad x, y \in B .
$$

Corollary 3. Let $A$ be a closed subspace of a commutative $C^{*}$-algebra $B$. A is a $C^{*}$-subalgebra if and only if with each $x$ in $A$ the element $\min (1,|x|)$ is in $A$ too.

Corollary 3 is an improvement of Proposition in Dellacherie [2, p. 52]. The following result is due to the referee. The result should be compared with $R$. V. Kadison [4, Theorem 6, p. 499].

Corollary 4. A continuous linear map $\Lambda$ between two $C^{*}$-algebras $B$ and $B^{\prime}$ is a $C^{*}$-algebra homomorphism if and only if

$$
\Lambda(|x|+1-|| x|-1|)=|\Lambda(x)|+1-|| \Lambda(x)|-1|
$$

for each $x$ in $B$.

Corollary 5. Let $A$ be a $C^{*}$-subalgebra of $B$. Assume that $B$ has identity 1. Then $A$ contains 1 if and only if, for some $x$ in $A, x^{-1}$ exists in $B$.

Proof. For the sufficiency part we notice that $|x|^{-1}$ exists whenever $x^{-1}$ exists. Hence let $x$ in $A$ be an element which is invertible in $B$. Then there is $\delta>0$ such that $|x| \geqslant \delta$. Hence $2 \delta=|x|+\delta-|| x|-\delta|$ is in $A$.

Corollary 6. Let $A$ be a vector space of real-valued functions. Assume that a real-valued function $f$ is in $A$ whenever there exists a sequence $\left\{f_{n} ; n \in \mathbf{N}\right\}$ in $A$ such that

$$
\lim _{n \rightarrow \infty} \sup _{x \in\{|f|<m\}}\left|f(x)-f_{n}(x)\right|=0
$$

for each positive $m$. Then $A$ is an algebra for the pointwise operations if and only if $A$ is a Stone lattice.

Here a Stone lattice $A$ is a vector space of functions for which $\min (1, f)$ is in $A$ for each $f$ in $A$; see H. Bauer [1, p. 194].

The author is indebted to the referee for suggesting some improvements in an earlier version of this paper. 
Thanks are due to the University of Antwerp and NFWO for their material support.

\section{REFERENCES}

1. H. Bauer, Probability theory and elements of measure theory, Holt, Rinehart and Winston, New York, 1972.

2. Cl. Dellacherie, Un complément au théorème de Stone-Weierstrass, Lecture Notes in Math., vol. 39, Springer-Verlag, Berlin, 1967, pp. 52-53. MR 37 \#4584.

3. J. Dixmier, Les $C^{*}$-algèbres et leurs représentations, Gauthier-Villars, Paris, 1969. MR 39 \#7442.

4. R.V. Kadison, A generalized Schwarz inequality and algebraic invariants for operator algebras, Ann. of Math. (2) 56 (1952), 494-503. MR 14, 481.

UNIVERSITEIT ANTWERPEN, ANTWERPEN, BELGIUM

Current address: Department of Mathematics, Universitaire Instelling, Antwerpen, Universiteitsplein 1, B-2610 Wilrijk, Belgium 